\title{
Optimality Conditions for a Nonsmooth Uncertain Multiobjective Programming Problem
}

\author{
Wenyan Han, Guolin Yu $\mathbb{D}$, and Tiantian Gong \\ Institute of Applied Mathematics, North Minzu University, Yinchuan 750021, China \\ Correspondence should be addressed to Guolin Yu; guolin_yu@126.com
}

Received 13 April 2020; Accepted 1 July 2020; Published 25 July 2020

Academic Editor: Sigurdur F. Hafstein

Copyright (C) 2020 Wenyan Han et al. This is an open access article distributed under the Creative Commons Attribution License, which permits unrestricted use, distribution, and reproduction in any medium, provided the original work is properly cited.

\begin{abstract}
This note is devoted to the investigation of optimality conditions for robust approximate quasi weak efficient solutions to a nonsmooth uncertain multiobjective programming problem (NUMP). Firstly, under the extended nonsmooth MangasarianFromovitz constrained qualification assumption, the optimality necessary conditions of robust approximate quasi weak efficient solutions are given by using an alternative theorem. Secondly, a class of generalized convex functions is introduced to the problem (NUMP), which is called the pseudoquasi-type-I function, and its existence is illustrated by a concrete example. Finally, under the pseudopseudo-type-I generalized convexity hypothesis, the optimality sufficient conditions for robust approximate quasi weak efficient solutions to the problem (NUMP) are established.
\end{abstract}

\section{Introduction}

It is well known that multiobjective programming problems are widely used in the fields of portfolio, resource allocation, and information transfer. In practical problems, most of the objectives or constraints to the optimization model are nonsmooth and are affected by various factors with uncertain information. Therefore, it is a very valuable work to study the nonsmooth uncertain optimization problems. Robust optimization is one of the effective methods to deal with uncertain optimization problems. The robust method is committed to ensuring the worst-case solution which is immunized against the data uncertainty to optimization problems, and for its more details, the reader is referred to [1]. In this paper, the optimality conditions to the nonsmooth uncertain multiobjective programming problem (NUMP) are described by using the robust optimization method.

Convexity and its generalization play an important role in mathematical programming, especially in establishing optimality sufficient conditions of optimization problems. Chuong and Kim [2] presented the generalized convex-affine function based on the Mordukhovich subdifferential for a class of nonsmooth multiobjective fractional programming problems. For objective and constraint functions $(f, g)$ of a class of nonsmooth robust multiobjective programming problems, the concept that $(f, g)$ is a generalized convexity of degree $n$ is introduced in literature [3]. Inspired by the generalized convexity in the above literatures, this paper introduces a kind of generalized convexities based on the Clarke subdifferential, which is called the $(f, g)$-pseudoquasi-type-I function, and under its assumption, it proves the optimality conditions of the problem (NUMP).

As we all know, the (weak) efficient solution of multiobjective optimization problems usually does not exist in the noncompact case, but the approximate solution exists under very mild conditions. In addition, most of the solutions obtained by the numerical algorithm are approximate solutions in the real world. Therefore, there exist the important theoretical value and practical significance to study the approximate solution of the optimization problem. Recently, Lee and Jiao [4] dealt with the optimality conditions of the robust approximate solution for an uncertain convex optimization problem involving a kind of constraint qualifications, which is called closed convex cone constrained qualification; Sun [5] established the optimality conditions of the robust optimal solution under the constrained qualification with respect to the subdifferential of the convex function; Sun and Li [6] discussed the optimality conditions 
of the robust approximate weak efficient solution under the hypothesis of closed convex cone constrained qualification. It is worth mentioning that the approximate weak efficient solution is a special case of the approximate quasi weak efficient solution. The purpose of this paper is to study optimality conditions of the robust approximate quasi weak efficient solution for the problem (NUMP). The convexities and constrained qualification are different from those of mentioned literatures, and we adopt the newly introduced $(f, g)$-pseudopseudo-type-I convexity and extended nonsmooth Mangasarian-Fromovitz constrained qualification (see [7]).

The content of this paper is arranged as follows: In Section 2, some basic concepts and lemmas which will be used in subsequent sections are proposed. The concept of the $(f, g)$-pseudoquasi-type-I generalized convexity with respect to the Clarke subdifferential is introduced, and an example is given to illustrate its existence. The main results are present in Section 3, in which the optimality conditions of the robust approximate quasi weak efficient solution to the problem (NUMP) are proven.

\section{Preliminaries}

This paper considers the following nonsmooth multiobjective programming problem (NMP):

$$
(\mathrm{NMP}) \begin{cases}\min & f(x)=\left(f_{1}(x), f_{2}(x), \ldots, f_{l}(x)\right), \\ \text { s.t. } & x \in C, g_{j}(x) \leq 0, \quad j=1, \ldots, m,\end{cases}
$$

where $C$ is a nonempty subset of $n$ dimension Euclid space $\mathbb{R}^{n}$ and $f_{i}, g_{j}: \mathbb{R}^{n} \longrightarrow \mathbb{R}, i=1, \ldots, l, j=1, \ldots, m$, are the Lipschitz functions. The feasible set of the problem (NMP) is denoted as

$$
\mathscr{F}_{0}:=\left\{x \in C: g_{j}(x) \leq 0, j=1, \ldots, m\right\} .
$$

When the constraint set of problem (NMP) contains uncertain data, the corresponding nonsmooth uncertain multiobjective programming problem (NUMP) is expressed as

$$
(\mathrm{NUMP}) \begin{cases}\min & f(x)=\left(f_{1}(x), f_{2}(x), \ldots, f_{l}(x)\right), \\ \text { s.t. } & x \in C, g_{j}\left(x, v_{j}\right) \leq 0, \quad j=1, \ldots, m,\end{cases}
$$

where $v_{j} \in \mathscr{V}_{j} \subset \mathbb{R}^{q}$ is the uncertain parameter, $\mathscr{V}_{j}$ is the compact convex set, and $g_{j}: \mathbb{R}^{n} \times \mathbb{R}^{q} \longrightarrow \mathbb{R}, j=1, \ldots, m$, are the Lipschitz functions with respect to the first variable. We denote $v=\left(v_{1}, \ldots, v_{m}\right) \in \mathscr{V}=\left(\mathscr{V}_{1}, \ldots, \mathscr{V}_{m}\right)$. The feasible set of problem (NUMP) is defined by

$$
\mathscr{F}_{v}:=\left\{x \in C: g_{j}\left(x, v_{j}\right) \leq 0, j=1, \ldots, m\right\} .
$$

The optimality conditions of problem (NUMP) will be studied by the robust optimization method in this note. For this purpose, we consider the following robust counterpart (see [8]) of the problem (NUMP):
$(\mathrm{NRMP}) \begin{cases}\min & f(x)=\left(f(x), f_{2}(x), \ldots, f(x)\right), \\ \text { s.t. } & x \in C, g_{j}\left(x, v_{j}\right) \leq 0, \forall v_{j} \in \mathscr{V}_{j}, \quad j=1, \ldots, m .\end{cases}$

The robust counterpart problem is called as the nonsmooth robust multiobjective programming problem (NRMP), and the robust feasible set of problem (NRMP) is given by

$$
\mathscr{F}:=\bigcap_{v \in \mathscr{V}} \mathscr{F}_{v} .
$$

Let

$\psi_{j}(x):=\max \left\{g_{j}\left(x, v_{j}\right): v_{j} \in \mathscr{V}_{j}\right\}, \quad j=1, \ldots, m, \forall x \in \mathbb{R}^{n}$.

For a given $\bar{x} \in \mathbb{R}^{n}$, we divide $J=\{1, \ldots, m\}$ into two index sets, $J=J_{1}(\bar{x}) \cup J_{2}(\bar{x})$, where

$$
\begin{aligned}
& J_{1}(\bar{x})=\left\{j \in J: \psi_{j}(\bar{x})=0\right\}, \\
& J_{2}(\bar{x})=\frac{J}{J_{1}(\bar{x})} .
\end{aligned}
$$

For any $j \in J_{1}(\bar{x})$, let

$$
\mathscr{V}_{j}(\bar{x}):=\left\{v_{j} \in \mathscr{V}_{j}: g_{j}\left(\bar{x}, v_{j}\right)=\psi_{j}(\bar{x})\right\}
$$

Let $\mathbb{B}$ be a closed unit ball in $\mathbb{R}^{n}$. For any $x, y \in \mathbb{R}^{n}$, use $\langle x, y\rangle$ to represent the inner product between $x$ and $y$. Set

$$
\begin{aligned}
\mathbb{R}_{+}^{n} & =\left\{x \in \mathbb{R}^{n}: x_{i} \geq 0,\right. & & (i=1, \ldots, n)\}, \\
\mathbb{R}_{++}^{n} & =\left\{x \in \mathbb{R}^{n}: x_{i}>0,\right. & & \left.J_{1}(\bar{x})\right\} .
\end{aligned}
$$

It is said that $\varphi: \mathbb{R}^{n} \longrightarrow \mathbb{R}$ is a convex function, if for any $x, y \in \mathbb{R}^{n}, \lambda \in[0,1]$,

$$
\varphi(\lambda x+(1-\lambda) y) \leq \lambda \varphi(x)+(1-\lambda) \varphi(y) .
$$

If $-\varphi$ is a convex function, then is said to be a concave function. For any $x \in \mathbb{R}^{n}$, if

$$
\limsup _{y \longrightarrow x} \varphi(y) \leq \varphi(x)
$$

then $\varphi$ is termed to be a upper semicontinuous function. Let $X \subset \mathbb{R}^{n}$ be a nonempty open subset. It is said that $\varphi: X \longrightarrow \mathbb{R}$ is a Lipschitz function, if there exists $L>0$, such that

$$
\left\|\varphi\left(x_{1}\right)-\varphi\left(x_{2}\right)\right\| \leq L\left\|x_{1}-x_{2}\right\|, \quad \forall x_{1}, x_{2} \in X .
$$

Let $d \in \mathbb{R}^{n}$, and the directional derivative (see [9]) of $\varphi$ at $\bar{x} \in X$ in the direction $d$ is given by

$$
\varphi^{\prime}(\bar{x} ; d)=\lim _{t \longrightarrow 0^{+}} \frac{\varphi(\bar{x}+t d)-\varphi(\bar{x})}{t},
$$

and the Clarke generalized directional derivative (see [9]) of $\varphi$ at $\bar{x}$ in the direction $d$ is defined by

$$
\varphi^{\circ}(\overline{\mathrm{x}} ; \mathrm{d})=\limsup _{\mathrm{y} \longrightarrow \overline{\mathrm{x}} \mathrm{t} \longrightarrow 0^{+}} \frac{\varphi^{\prime}(\mathrm{y}+\mathrm{td})-\varphi(\mathrm{y})}{\mathrm{t}},
$$


If

$$
\varphi^{\circ}(\overline{\mathrm{x}} ; \mathrm{d})=\varphi \prime(\overline{\mathrm{x}} ; \mathrm{d}), \quad \forall \mathrm{d} \in \mathbb{R}^{\mathrm{n}},
$$

then $\varphi$ is called to be regular at $\bar{x}$. The Clarke subdifferential (see [9]) of $\varphi$ at $\bar{x}$ is denoted as

$$
\partial \varphi(\bar{x}):=\left\{\xi \in \mathbb{R}^{n}: \varphi^{\circ}(\overline{\mathrm{x}} ; \mathrm{d}) \geq\langle\xi, \mathrm{d}\rangle, \forall \mathrm{d} \in \mathbb{R}^{\mathrm{n}}\right\} .
$$

Lemma 1 (see [7]). Let $\varphi: X \subset \mathbb{R}^{n} \longrightarrow \mathbb{R}$ be a Lipschitz function, then the following conclusions hold:

(i) $\partial \varphi(x)$ is a nonempty compact convex set in $X$, and for any $\xi \in \partial \varphi(x)$, one has $\|\xi\| \leq L$ ( $L$ is Lipschitz constant of $\varphi$ ).

(ii) $d \mapsto \varphi^{\circ}(x ; d)$ is convex, and

$$
\left|\varphi^{\circ}(\mathrm{x} ; \mathrm{d})\right| \leq \mathrm{L}\|\mathrm{d}\| .
$$

(iii) For any $d \in \mathbb{R}^{n}$, we have

$$
\varphi^{\circ}(\mathrm{x} ; \mathrm{d})=\max \{\langle\xi, \mathrm{d}\rangle: \xi \in \partial \varphi(\mathrm{x})\} .
$$

For a given function $g: U \times \mathscr{V}_{0} \subset \mathbb{R}^{n} \times \mathbb{R}^{q} \longrightarrow \mathbb{R}$, suppose $g$ satisfies the following assumptions (see [7]):

(i) $g\left(x, v_{0}\right)$ is upper semicontinuous in $\left(x, v_{0}\right) \in U \times$ $\mathscr{V}_{0}$.

(ii) $g\left(x, v_{0}\right)$ is a Lipschitz function with respect to the first variable $x \in U$, that is, $g\left(\cdot, v_{0}\right)$ is a Lipschitz function for any $v_{0} \in \mathscr{V}_{0}$.

(iii) $g$ is regular with respect to the first variable $x \in U$, that is,

$$
g_{x}^{\circ}\left(x, v_{0} ; \cdot\right)=g_{x}^{\prime}\left(x, v_{0} ; \cdot\right)
$$

where ${ }_{g}^{x^{\circ}}\left(x, v_{0} ; \cdot\right)$ is a Clarke generalized directional derivative with respect to the first variable $x$ and $g_{x}^{\prime}\left(x, v_{0} ; \cdot\right)$ is the directional derivative with respect to the first variable $x$.

(iv) The Clarke subdifferential $\partial_{x} g\left(x, v_{0}\right)$ with respect to the first variable $x$ is weak * upper semicontinuous in $\left(x, v_{0}\right) \in U \times \mathscr{V}_{0}$.

Let

$$
\psi(x):=\max \left\{g\left(x, v_{0}\right): v_{0} \in \mathscr{V}_{0}\right\}, \quad \forall x \in \mathbb{R}^{n} .
$$

If $g\left(x, v_{0}\right)$ satisfies Assumptions (i)-(iv), then $\psi(x)$ is a Lipschitz function (see [7]). For a given $\bar{x} \in \mathbb{R}^{n}$, set

$$
\mathscr{V}_{0}(\bar{x}):=\left\{v_{0} \in \mathscr{V}_{0}: g\left(\bar{x}, v_{0}\right)=\psi(\bar{x})\right\} .
$$

Remark 1. (see [7]). If Assumptions (i)-(iv) are fufilled, $\mathscr{V}_{0}$ is a convex subset and $g(x, \cdot)$ is a concave on $\mathscr{V}_{0}$; then,

$$
\partial \psi(x)=\left\{\xi: \exists v_{0} \in \mathscr{V}_{0}(x) \text { s.t. } \xi \in \partial_{x} g\left(x, v_{0}\right)\right\}, \quad \forall x \in \mathbb{R}^{n} .
$$

Lemma 2 (see [9]). Let $\varphi, \phi: X \subset \mathbb{R}^{n} \longrightarrow \mathbb{R}$ be a Lipschitz function, $\bar{x} \in X$. Then,

$$
\partial(\varphi+\phi)(\bar{x}) \subset \partial \varphi(\bar{x})+\partial \phi(\bar{x})
$$

If $\varphi, \phi$ are regular at $\bar{x}$, then $\varphi+\phi$ is regular at $\bar{x}$, and $\partial(\varphi+\phi)(\bar{x})=\partial \varphi(\bar{x})+\partial \phi(\bar{x})$.

Lemma 3 (alternative theorem, see [10]). Let $A \subset \mathbb{R}^{n}$ be a convex set, and $\phi_{1}(x), \ldots, \phi_{m}(x)$ are convex on $A$, if the following system of inequalities

$$
\phi(x)<0, \quad i=1, \ldots, m, \forall x \in A,
$$

has no solution on $A$, then there exist $\lambda_{1}, \ldots, \lambda_{m} \geq 0$, not all zero, such that

$$
\sum_{i=1}^{m} \lambda_{i} \phi_{i}(x) \geq 0, \quad \forall x \in A .
$$

Definition 1 (see [3]). Let $\varepsilon=\left(\varepsilon_{1}, \ldots, \varepsilon_{l}\right) \in \mathbb{R}_{+}^{l}, \bar{x} \in \mathscr{F}$.

(i) It is said that $\bar{x}$ is a robust $\varepsilon$-quasi weak efficient solution of the problem (NUMP), iff $\bar{x}$ is an $\varepsilon$-quasi weak efficient solution of the problem (NRMP), that is,

$$
(f(x)-f(\bar{x})+\sqrt{\varepsilon}\|x-\bar{x}\|) \notin-\mathbb{R}_{++}^{l}, \quad \forall x \in \mathscr{F} .
$$

(ii) It is called that $\bar{x}$ is a robust $\varepsilon$-quasi efficient solution of the problem (NUMP), iff $\bar{x}$ is an $\varepsilon$-quasi efficient solution of the problem (NRMP), that is,

$$
(f(x)-f(\bar{x})+\sqrt{\varepsilon}\|x-\bar{x}\|) \notin-\frac{\mathbb{R}_{+}^{l}}{\{0\}}, \quad \forall x \in \mathscr{F} .
$$

The following generalized convexity is introduced for the objective and constraint functions $(f, g)$ to the problem (NUMP).

Definition 2. It is said that $(f, g)$ is a pseudoquasi-type-I function at $\bar{x} \in C$, if for any $x \in C, \quad \xi_{i} \in \partial$ $f_{i}(\bar{x}), \quad i=1, \ldots, l, b \in \mathbb{B}, \quad \eta_{j} \in \partial_{x} g_{j}\left(\bar{x}, v_{j}\right)$, and $v_{j} \in$ $\mathscr{V}_{j}(\bar{x}), j=1, \ldots, m$, there exists $u \in \mathbb{R}^{n}$, such that

$$
\begin{array}{r}
\left\langle\xi_{i}, u\right\rangle+\sqrt{\varepsilon_{i}}\langle b, u\rangle \geq 0 \Longrightarrow f_{i}(x)-f_{i}(\bar{x})+\sqrt{\varepsilon_{i}}\|x-\bar{x}\| \geq 0, \\
i=1, \ldots, l,
\end{array}
$$

$g_{j}\left(x, v_{j}\right) \leq g_{j}\left(\bar{x}, v_{j}\right) \Longrightarrow\left\langle\eta_{j}, u\right\rangle \leq 0, \quad j=1, \ldots, m$.

If equation (29) takes a strictly inequality, i.e., 


$$
\begin{array}{r}
\left\langle\xi_{i}, u\right\rangle+\sqrt{\varepsilon_{i}}\langle b, u\rangle \geq 0 \Longrightarrow f_{i}(x)-f_{i}(\bar{x})+\sqrt{\varepsilon_{i}} \| x-\bar{x}>0, \\
i=1, \ldots, l,
\end{array}
$$

then $(f, g)$ is called a strictly pseudoquasi-type-I function at $\bar{x} \in C$.

The following is an example to illustrate the existence of the pseudoquasi-type-I function.

Example 1. Let $f_{i}: \mathbb{R} \longrightarrow \mathbb{R}, i=1,2$, be given by

$$
\begin{aligned}
& f_{1}(x)= \begin{cases}\frac{3}{2} x, & \text { if } x \geq 0, \\
x, & \text { if } x<0,\end{cases} \\
& f_{2}(x)= \begin{cases}\frac{4}{3} x, & \text { if } x \geq 0, \\
x, & \text { if } x<0,\end{cases}
\end{aligned}
$$

and $g: \mathbb{R} \times \mathscr{V} \longrightarrow \mathbb{R}$ be defined as

$$
g(x, v)=v x^{2}, x \in \mathbb{R}, v \in \mathscr{V} \backslash\{0\}, g(x, 0)= \begin{cases}-\frac{2}{3} x, & \text { if } x<0, \\ -x, & \text { if } x \geq 0,\end{cases}
$$

where $\mathscr{V}=[0,1]$. Taking $\bar{x}=0, \sqrt{\varepsilon}=\left(\sqrt{\varepsilon}_{1}, \sqrt{\varepsilon}_{2}\right)=(1,1)$, by simple calculation, we can obtain that $\partial f_{1}(\bar{x})=$ $[1,3 / 2], \partial f_{2}(\bar{x})=[1,4 / 3], \quad \partial_{x} g(\bar{x}, v)=\{0\}, \quad v \in \mathscr{V} \backslash\{0\}$, $\partial_{x} g(\bar{x}, 0)=[-1,-2 / 3]$, and $\mathscr{V}(\bar{x})=[0,1] \sqrt{2}$. For any $x \in \mathbb{R}, \xi_{i} \in \partial f_{i}(\bar{x}), i=1,2, b \in \mathbb{B}, \eta \in \partial_{x} g(\bar{x}, 0)$, and $v \in$ $\mathscr{V}(\bar{x})$, there exists $0 \leq u \in \mathbb{R}$, such that

$$
\begin{aligned}
& \left\langle\xi_{1}, u\right\rangle+\sqrt{\varepsilon_{1}}\langle b, u\rangle=\left\langle\xi_{1}, u\right\rangle+\langle b, u\rangle \geq 0 \Longrightarrow f_{1}(x)-f_{1}(\bar{x}) \\
& +\sqrt{\varepsilon_{1}}\|x-\bar{x}\|=f_{1}(x)+\|x\| \geq 0
\end{aligned}
$$

$$
\begin{aligned}
& \left\langle\xi_{2}, u\right\rangle+\sqrt{\varepsilon_{2}}\langle b, u\rangle=\left\langle\xi_{2}, u\right\rangle+\langle b, u\rangle \geq 0 \Longrightarrow f_{2}(x)-f_{2}(\bar{x}) \\
& +\sqrt{\varepsilon_{2}}\|x-\bar{x}\|=f_{2}(x)+\|x\| \geq 0 \\
& g(x, v) \leq g(\bar{x}, v) \Longrightarrow 0 \cdot u \leq 0, \quad v \in \mathscr{V} \backslash\{0\} \\
& g(x, 0) \leq g(\bar{x}, 0) \Longrightarrow \eta \cdot u \leq 0, \quad v=0 .
\end{aligned}
$$

Hence, $(f, g)$ is a pseudoquasi-type-I function at $\bar{x}=0$.

\section{Optimality Conditions}

In this section, we begin with establishing the optimality necessary conditions for a robust $\mathcal{\varepsilon}$-quasi weak efficient solution to the problem (NUMP) by using the alternative theorem (Lemma 3).

Theorem 1. In the problem (NUMP), if $\bar{x} \in \mathscr{F}$ is a robust $\varepsilon$-quasi weak efficient solution of the problem (NUMP), then there exists not all the zero real values $\bar{\lambda}_{i} \geq 0, i=1, \ldots, l, \bar{\mu}_{j} \geq 0, j \in J_{1}(\bar{x})$, such that

$$
\begin{aligned}
& \sum_{i=1}^{l} \bar{\lambda}_{i} f_{i}^{\circ}(\bar{x} ; d)+\sum_{j \in J_{1}(\bar{x})} \bar{\mu}_{j} \psi_{j}^{\circ}(\bar{x} ; d) \\
& +\sum_{i=1}^{l} \bar{\lambda}_{i}\left(\sqrt{\varepsilon}_{i}\|\cdot-\bar{x}\|\right)^{\circ}(\bar{x} ; d) \geq 0, \quad \forall d \in \mathbb{R}^{n} .
\end{aligned}
$$

Proof. Firstly, we claim that the following system of inequalities:

$$
\left\{\begin{array}{l}
f_{i}^{\circ}(\bar{x}, d)+\left(\sqrt{\varepsilon}_{i}\|\cdot-\bar{x}\|\right)^{\circ}(\bar{x}, d)<0, \quad i=1, \ldots, l, \\
\psi_{j}^{\circ}(\bar{x}, d)<0, \quad \forall j \in J_{1}(\bar{x}),
\end{array}\right.
$$

has no solution $d ı \in \mathbb{R}^{n}$. Otherwise, there exists $d \in \mathbb{R}^{n}$, such that

$$
\begin{aligned}
f_{i}^{\circ}(\bar{x}, d)+\left(\sqrt{\varepsilon}_{i}\|\cdot-\bar{x}\|\right)^{\circ}(\bar{x}, d)<0, & i=1, \ldots, l, \\
\psi_{j}^{\circ}(\bar{x}, d)<0, & \forall j \in J_{1}(\bar{x}) .
\end{aligned}
$$

Since

$$
\underset{t \longrightarrow 0^{+}}{\limsup } \frac{f_{i}(\bar{x}+t d)-f_{i}(\bar{x})}{t}=\inf _{\bar{\delta}_{i}^{1}>0} \sup _{\substack{0<t<\bar{\delta}_{i}^{1} \\ s_{i}}} \frac{f_{i}(\bar{x}+t d)(\bar{x}, d)}{t},
$$

$$
\begin{gathered}
\limsup _{t \longrightarrow 0^{+}} \frac{\sqrt{\varepsilon_{i}}\|\bar{x}+t d-\bar{x}\|-\sqrt{\varepsilon} i\|\bar{x}-\bar{x}\|}{t} \\
=\inf _{\bar{\delta}_{i}^{2}>0} \sup _{0<t<\bar{\delta}_{i}^{2}} \frac{\sqrt{\varepsilon_{i}}\|\bar{x}+t d-\bar{x}\|-\sqrt{\varepsilon} i\|\bar{x}-\bar{x}\|}{t}, \\
i=1, \ldots, l,
\end{gathered}
$$


we get

$$
\begin{aligned}
& \limsup _{t \rightarrow 0^{+}}\left(\frac{f_{i}(\bar{x}+t d)+\sqrt{\varepsilon}_{i}\|\bar{x}+t d-\bar{x}\|-f_{i}(\bar{x})-\sqrt{\varepsilon}{ }_{i}\|\bar{x}-\bar{x}\|}{t}\right) \\
& \leq \limsup _{t \longrightarrow 0^{+}} \frac{f_{i}(\bar{x}+t d)-f_{i}(\bar{x})}{t}+\limsup _{t \longrightarrow 0^{+}} \frac{\sqrt{\varepsilon_{i}}\|\bar{x}+t d-\bar{x}\|-\sqrt{\varepsilon_{i}}\|\bar{x}-\bar{x}\|}{t} \\
& =\inf _{\bar{\delta}_{i}^{1}>0} \sup _{0<t<\bar{\delta}_{i}^{1}} \frac{f_{i}(\bar{x}+t d)-f_{i}(\bar{x})}{t}+\inf _{\bar{\delta}_{i}^{2}>0} \sup _{0<t<\bar{\delta}_{i}^{2}} \frac{\sqrt{\varepsilon_{i}}\|\bar{x}+t d-\bar{x}\|-\sqrt{\varepsilon_{i}}\|\bar{x}-\bar{x}\|}{t} \\
& \leq \inf _{\bar{\delta}_{i}^{1}>0 €>0} \sup _{0<\epsilon<\bar{\delta}_{i}^{1}\|h\|<\epsilon} \frac{f_{i}(\bar{x}+h+t d)-f_{i}(\bar{x}+h)}{t}+ \\
& \inf _{\bar{\delta}_{i}^{2}>0 €>0} \sup _{0<t<\bar{\delta}_{i}^{2}\|h\|<\epsilon} \frac{\sqrt{\varepsilon_{i}}\|\bar{x}+h+t d-\bar{x}\|-\sqrt{\varepsilon} i\|\bar{x}+h-\bar{x}\|}{t} \\
& =\limsup _{\mathrm{h} \longrightarrow 0, \mathrm{t} \longrightarrow 0^{+}} \frac{f_{i}(\bar{x}+h+t d)-f_{i}(\bar{x}+h)}{t}+\limsup _{\mathrm{h} \longrightarrow 0, \mathrm{t} \longrightarrow 0^{+}} \frac{\sqrt{\varepsilon_{i}}\|\bar{x}+h+t d-\bar{x}\|-\sqrt{\varepsilon_{i}}\|\bar{x}+h-\bar{x}\|}{t} \\
& =f_{i}^{\circ}(\bar{x}, d)+\left(\sqrt{\varepsilon}_{i}\|\cdot-\bar{x}\|\right)^{\circ}(\bar{x}, d)<0, \quad i=1, \ldots, l .
\end{aligned}
$$

Therefore, there exists $\bar{\delta}_{i}^{*}$, for any $0<t<\bar{\delta}_{i}^{*}$, and we arrive at

$$
f_{i}(\bar{x}+t d)-f_{i}(\bar{x})+\sqrt{\varepsilon}_{i}\|\bar{x}+t d-\bar{x}\|<0, \quad i=1, \ldots, l .
$$

On the other hand, for any $j \in J_{1}(\bar{x})$,

$$
\begin{aligned}
& \limsup _{t \rightarrow 0^{+}} \frac{\psi_{j}(\bar{x}+t d)-\psi_{j}(\bar{x})}{t}=\inf _{\tilde{\delta}_{j}^{1}>0} \sup _{0<t<\widetilde{\delta}_{j}^{-1}} \frac{\psi_{j}(\bar{x}+t d)-\psi_{j}(\bar{x})}{t} \\
& \leq \inf _{\substack{\tilde{\delta}_{j}>0 \varepsilon>0 \\
0<<<<\delta_{j}^{1}\|h\|<\varepsilon}} \sup _{0,1} \frac{\psi_{j}(\bar{x}+h+t d)-\psi_{j}(\bar{x}+h)}{t} \\
& =\limsup _{h \longrightarrow 0, t \rightarrow 0^{+}} \frac{\psi_{j}(\bar{x}+h+t d)-\psi_{j}(\bar{x}+h)}{t}={ }_{\psi}^{j^{\circ}}(\bar{x} ; d)<0 .
\end{aligned}
$$

Hence, there exists $\widetilde{\delta}_{j}^{*}, j \in J_{1}(\bar{x})$ such that $\psi_{j}(\bar{x}+t d)<\psi_{j}(\bar{x})=0$, for any $0<t<\delta_{j}^{*}$. In addition, for any $j \in J_{2}(\bar{x})$, one has $\psi_{j}(\bar{x})<0$. Noticing that $\psi_{j}$ is a Lipschitz function, we know that there exists $\widehat{\delta}_{j}^{*}>0$, for any $t \in\left(0, \widehat{\delta}_{j}^{*}\right)$ :

$$
\psi_{j}(\bar{x}+t d)<0, \forall j \in J_{2}(\bar{x}) .
$$

Let $\delta^{*}=\min _{\widetilde{\delta}^{*}}\left(\bar{\delta}^{*}, \widetilde{\delta}^{*}, \widehat{\delta}^{*}\right)$, where $\bar{\delta}^{*}=\min _{i \in\{1, \ldots, l\}} \bar{\delta}_{i}^{*}$, $\widetilde{\delta}^{*}=\min _{j \in J_{1}(\bar{x})} \widetilde{\delta}_{j}^{*}$, and $\widehat{\delta}^{*}=\min _{j \in J_{2}(\bar{x})} \widehat{\delta}_{j}^{*}$. Then, for any $t \in\left(0, \delta^{*}\right)$, it yields that

$$
\begin{array}{r}
\bar{x}+t d \in \mathscr{F}, f_{i}(\bar{x}+t d)-f_{i}(\bar{x})+\sqrt{\varepsilon_{i}}\|\bar{x}+t d-\bar{x}\|<0, \\
i=1, \ldots, l,
\end{array}
$$

which contradicts to the fact that $\bar{x} \in \mathscr{F}$ is a robust $\varepsilon$-quasi weak efficient solution of the problem (NUMP).

We conclude from Lemma 1 (ii) that

$$
\begin{gathered}
d \longmapsto f_{i}^{\circ}(\bar{x} ; d)+\left(\sqrt{\varepsilon}\left\|_{i} \cdot-\bar{x}\right\|\right)^{\circ}(\bar{x} ; d), \quad i=1, \ldots, l, \\
\psi_{j}^{\circ}(\bar{x} ; d), \quad j \in J_{1}(\bar{x})
\end{gathered}
$$

are convex functions. Again by Lemma 3, it can be known that there exists not all zero real values $\bar{\lambda}_{i} \geq 0, \quad i=1, \ldots, l, \bar{\mu}_{j} \geq 0, j \in J_{1}(\bar{x})$, such that equation (38) holds.

Next, we will examine the optimality necessary conditions of the robust $\varepsilon$-quasi weak efficient solution to the problem (NUMP). For this purpose, we need to introduce the following extended nonsmooth Mangasarian-Fromovitz constrained qualification.

Definition 3. In the problem (NRMP), let $\bar{x} \in \mathscr{F}$. If

$$
\exists d \in \mathbb{R}^{n} \quad \text { s.t. } g_{j x}^{\circ}\left(\bar{x}, v_{j} ; d\right)<0, \forall v_{j} \in \mathscr{V}_{j}(\bar{x}), j \in J_{1}(\bar{x}),
$$

then it is called that the problem (NRMP) satisfies extended the nonsmooth Mangasarian-Fromovitz constrained qualification at $\bar{x}$.

Theorem 2. In the problem (NUMP), suppose that $g_{j}, j=1, \ldots, m$, satisfy Assumptions (i)-(iv), and for any $x \in \mathbb{R}^{n}, g_{j}(x, \cdot)$ is a concave function on $\mathscr{V}_{j}$. If $\bar{x} \in \mathscr{F}$ is a robust $\mathcal{\varepsilon}$-quasi weak efficient solution to the problem $(N U M P)$, then there exists $(\bar{\lambda}, \bar{\mu}, \bar{v}) \in \mathbb{R}_{+}^{l} \times \mathbb{R}_{+}^{m} \times \mathscr{V}$ and $(\bar{\lambda}, \bar{\mu}) \neq 0, \quad$ where $\bar{\lambda}=\left(\bar{\lambda}_{1}, \ldots, \bar{\lambda}_{l}\right), \quad \bar{\mu}=\left(\bar{\mu}_{1}, \ldots, \bar{\mu}_{m}\right)$, $\bar{v}=\left(\bar{v}_{1}, \ldots, \bar{v}_{m}\right)$, and $\bar{v}_{j} \in \mathscr{V}_{j}(\bar{x}), j=1, \ldots, m$, such that

$$
0 \in \sum_{i=1}^{l} \bar{\lambda}_{i} \partial f_{i}(\bar{x})+\sum_{j=1}^{m} \bar{\mu}_{j} \partial_{x} g_{j}\left(\bar{x}, \bar{v}_{j}\right)+\sum_{i=1}^{l} \bar{\lambda}_{i} \sqrt{\varepsilon_{i} \mathbb{B}},
$$

$$
\bar{\mu}_{j} g_{j}\left(\bar{x}, \bar{v}_{j}\right)=0, \quad j=1, \ldots, m .
$$


In addition, if the problem (NRMP) satisfies the extended nonsmooth Mangasarian-Fromovitz constrained qualification at $\bar{x}$, then there exists $\bar{\lambda} \in \mathbb{R}_{+}^{l} \backslash\{0\}$ and $\bar{\mu} \in \mathbb{R}_{+}^{m}, \bar{v}_{j} \in \mathscr{V}_{j}(\bar{x}), j=1, \ldots, m$, such that equations (52) and (53) hold.

Proof. Since $\bar{x}$ is a robust $\mathcal{\varepsilon}$-quasi weak efficient solution to the problem (NUMP), it yields from Theorem 2 that there exists not all zero real values $\bar{\lambda}_{i} \geq 0, i=1, \ldots, l$, $\bar{\mu}_{j} \geq 0, j \in J_{1}(\bar{x})$, such that

$$
\sum_{i=1}^{l} \bar{\lambda}_{i} f_{i}^{\circ}(\bar{x} ; d)+\sum_{j \in J_{1}(\bar{x})} \bar{\mu}_{j} \psi_{j}^{\circ}(\bar{x} ; d)+\sum_{i=1}^{l} \bar{\lambda}_{i}\left(\sqrt{\varepsilon}_{i}\|\cdot-\bar{x}\|\right)^{\circ},
$$$$
(\bar{x} ; d) \geq 0, \forall d \in \mathbb{R}^{n} .
$$

According to Lemma 1 (iii), we know that

$$
\begin{aligned}
& \sum_{i=1}^{l} \bar{\lambda}_{i} \max \left\{\left\langle\xi_{i}, d\right\rangle: \xi_{i} \in \partial f_{i}(\bar{x})\right\} \\
& \quad+\sum_{j \in J_{1}(\bar{x})} \bar{\mu}_{j} \max \left\{\left\langle\eta_{j}, d\right\rangle: \eta_{j} \in \partial \psi_{j}(\bar{x})\right\} \\
& \quad+\sum_{i=1}^{l} \bar{\lambda}_{i} \max \left\{\left\langle\zeta_{i}, d\right\rangle: \zeta_{i} \in \sqrt{\varepsilon}_{i} \mathbb{B}\right\} \geq 0, \quad \forall d \in \mathbb{R}^{n} .
\end{aligned}
$$

This means that

$$
\max _{\xi_{i} \in \partial f_{i}(\bar{x}) \eta_{j} \in \partial \psi_{j}(\bar{x}) \zeta_{i} \in \sqrt{\varepsilon_{i} \mathbb{B}}}\left\{\sum_{i=1}^{l} \bar{\lambda}_{i} \xi_{i}+\sum_{j \in J_{1}(\bar{x})} \bar{\mu}_{j} \eta_{j}+\sum_{i=1}^{l} \bar{\lambda}_{i} \zeta_{i}, d\right\} \geq 0,
$$

this is equivalent to

$$
\begin{aligned}
& \inf _{d \in \mathbb{B}} \max _{\xi_{i} \in \partial f_{i}(\bar{x})} \max _{j} \in \partial \psi_{j}(\bar{x}) \zeta_{i} \in \sqrt{\varepsilon_{i} \mathbb{B}} \\
& \cdot\left\{\sum_{i=1}^{l} \bar{\lambda}_{i} \xi_{i}+\sum_{j \in J_{1}(\bar{x})} \bar{\mu}_{j} \eta_{j}+\sum_{i=1}^{l} \bar{\lambda}_{i} \zeta_{i}, d\right\} \geq 0 .
\end{aligned}
$$

Noticing that $\partial f_{i}(\bar{x}), \sqrt{\varepsilon}_{i} \mathbb{B}, i=1, \ldots, l, \partial \psi_{j}(\bar{x})$, $j \in J_{1}(\bar{x})$, are the nonempty compact convex set in $\mathbb{R}^{n}$ (by Lemma 1 (i)). Hence, it follows from lop-sided minimax theorem [11] that there exists $\bar{\xi}_{i} \in \partial f_{i}(\bar{x}), \bar{\zeta}_{i} \in \sqrt{\varepsilon}_{i} \mathbb{B}$, $i=1, \ldots, l, \bar{\eta}_{j} \in \partial \psi_{j}(\bar{x}), j \in J_{1}(\bar{x})$, such that

$$
\begin{aligned}
& \inf _{d \in \mathbb{B}}\left\{\sum_{i=1}^{l} \bar{\lambda}_{i} \bar{\xi}_{i}+\sum_{j \in J_{1}(\bar{x})} \bar{\mu}_{j} \bar{\eta}_{i}+\sum_{i=1}^{l} \bar{\lambda}_{i} \bar{\zeta}_{i}, d\right\} \\
& =\max _{\xi_{i} \in \partial f_{i}(\bar{x})} \max _{j} \in \partial \psi_{j}(\bar{x}) \zeta_{i} \in \sqrt{\varepsilon_{i}} \mathbb{B} \\
& \quad \inf _{d \in \mathbb{B}}\left\{\sum_{i=1}^{l} \bar{\lambda}_{i} \xi_{i}+\sum_{j \in J_{1}(\bar{x})} \bar{\mu}_{j} \eta_{j}+\sum_{i=1}^{l} \bar{\lambda}_{i} \zeta_{i}, d\right\} \geq 0 .
\end{aligned}
$$

Again because

$$
\begin{aligned}
& \inf _{d \in \mathbb{B}}\left\{\sum_{i=1}^{l} \bar{\lambda}_{i} \bar{\xi}_{i}+\sum_{j \in J_{1}(\bar{x})} \bar{\mu}_{j} \bar{\eta}_{j}+\sum_{i=1}^{l} \bar{\lambda}_{i} \bar{\zeta}_{i}, d\right\} \\
& =-\left\|\sum_{i=1}^{l} \bar{\lambda}_{i} \bar{\xi}_{i}+\sum_{j \in J_{1}(\bar{x})} \bar{\mu}_{j} \bar{\eta}_{j}+\sum_{i=1}^{l} \bar{\lambda}_{i} \bar{\zeta}_{i}\right\| \geq 0,
\end{aligned}
$$

we arrive at

$$
\left\|\sum_{i=1}^{l} \bar{\lambda}_{i} \bar{\xi}_{i}+\sum_{j \in J_{1}(\bar{x})} \bar{\mu}_{j} \bar{\eta}_{j}+\sum_{i=1}^{l} \bar{\lambda}_{i} \bar{\zeta}_{i}\right\|=0 .
$$

Therefore,

$$
0 \in \sum_{i=1}^{l} \bar{\lambda}_{i} \partial f_{i}(\bar{x})+\sum_{j \in J_{1}(\bar{x})} \bar{\mu}_{j} \partial \psi_{j}(\bar{x})+\sum_{i=1}^{l} \bar{\lambda}_{i} \sqrt{\varepsilon_{i}} \mathbb{B} .
$$

In addition, for any $j \in J_{2}(\bar{x})$, let $\bar{\mu}_{j}=0$. Then, from the above equation, we get that

$$
\begin{gathered}
0 \in \sum_{i=1}^{l} \bar{\lambda}_{i} \partial f_{i}(\bar{x})+\sum_{j=1}^{m} \bar{\mu}_{j} \partial \psi_{j}(\bar{x})+\sum_{i=1}^{l} \bar{\lambda}_{i} \sqrt{\varepsilon_{i}} \mathbb{B}, \\
\bar{\mu}_{j} \psi_{j}(\bar{x})=0, \quad j=1, \ldots, m .
\end{gathered}
$$

It yields from Remark 1 that there exists $\bar{v}_{j} \in \mathscr{V}_{j}(\bar{x}), j=1, \ldots, m$, such that (52) and (53) are true.

On the other hand, if the problem (NRMP) satisfies the extended nonsmooth Mangasarian-Fromovitz constrained qualification at $\bar{x}$ and $\bar{\lambda}=0$, then there exists $\bar{\mu} \in \mathbb{R}_{+}^{m}$, $\bar{v}_{j} \in \mathscr{V}_{j}(\bar{x}), j \in J_{1}(\bar{x})$, such that

$$
0 \in \sum_{j \in J_{1}(\bar{x})} \bar{\mu}_{j} \partial_{x} g_{j}\left(\bar{x}, \bar{v}_{j}\right)=\partial_{x}\left(\sum_{j \in J_{1}(\bar{x})} \bar{\mu}_{j} g_{j}\left(\bar{x}, \bar{v}_{j}\right)\right),
$$

and the above equation holds according to Lemma 2. Again by Lemma 1 (iii), we have

$$
\sum_{j \in J_{1}(\bar{x})} \bar{\mu}_{j} g_{j x}^{\circ}\left(\bar{x}, v_{j} ; d\right) \geq 0, \quad \forall d \in \mathbb{R}^{n},
$$

which contradicts to Definition 3. Hence, this leads to $\bar{\lambda} \in \mathbb{R}_{+}^{l} \backslash\{0\}$.

It is said that equations (52) and (53) are robust optimality necessary conditions of the problem (NUMP). We present the following Theorem 3 which is an optimality sufficient condition for the robust $\varepsilon$-quasi weak efficient solution to the problem (NUMP).

Theorem 3. In the problem (NUMP), supposing that $(\bar{x}, \bar{\lambda}, \bar{\mu}, \bar{v}) \in \mathscr{F} \times\left(\mathbb{R}_{+}^{l} \backslash\{0\}\right) \times \mathbb{R}_{+}^{m} \times \mathscr{V}$ satisfies the robust optimality necessary conditions.

(i) If $(f, g)$ is a pseudoquasi-type-I function at $\bar{x}$, then $\bar{x}$ is a robust $\varepsilon$-quasi weak efficient solution of the problem (NUMP).

(ii) If $(f, g)$ is a strictly pseudoquasi-type-I function at $\bar{x}$, then $\bar{x}$ is a robust $\varepsilon$-quasi efficient solution of the problem (NUMP). 
Proof. By the given conditions, it follows that the robust optimality necessary conditions hold at $(\bar{x}, \bar{\lambda}, \bar{\mu}, \bar{v}) \in \mathscr{F} \times\left(\mathbb{R}_{+}^{l} \backslash\{0\}\right) \times \mathbb{R}_{+}^{m} \times \mathscr{V}$, that is,

$$
\begin{gathered}
0 \in \sum_{i=1}^{l} \bar{\lambda}_{i} \partial f_{i}(\bar{x})+\sum_{j=1}^{m} \bar{\mu}_{j} \partial_{x} g_{j}\left(\bar{x}, \bar{v}_{j}\right)+\sum_{i=1}^{l} \bar{\lambda}_{i} \sqrt{\varepsilon_{i}} \mathbb{B}, \\
\bar{\mu}_{j} g_{j}\left(\bar{x}, \bar{v}_{j}\right)=0, \quad j=1, \ldots, m .
\end{gathered}
$$

Therefore, there exists $\bar{\xi}_{i} \in \partial f_{i}(\bar{x}), b \in \mathbb{B}$, $\bar{\eta}_{j} \in \partial_{x} g_{j}\left(\bar{x}, \bar{v}_{j}\right), j=1, \ldots, m$, such that

$$
\sum_{i=1}^{l} \bar{\lambda}_{i} \bar{\xi}_{i}+\sum_{j=1}^{m} \bar{\mu}_{j} \bar{\eta}_{j}+\sum_{i=1}^{l} \bar{\lambda}_{i} \sqrt{\varepsilon_{i}} b=0 .
$$

Let us prove conclusion (i). If $\bar{x}$ is not a robust $\varepsilon$-quasi weak efficient solution of the problem (NUMP), then there exists $\widehat{x} \in \mathscr{F}$, such that

$$
f(\widehat{x})-f(\bar{x})+\sqrt{\varepsilon}\|\widehat{x}-\bar{x}\| \in-\mathbb{R}_{++}^{l},
$$

that is,

$$
f(\widehat{x})-f(\bar{x})+\sqrt{\varepsilon}\|\widehat{x}-\bar{x}\|<0, \quad i=1, \ldots, l .
$$

On the other hand, in equation (66), if $\bar{\mu}_{j} \neq 0$, then $g_{j}\left(\bar{x}, \bar{v}_{j}\right)=0, \quad j=1, \ldots, m$. Again since $\widehat{x} \in \mathscr{F}$, then

$$
g_{j}\left(\hat{x}, \bar{v}_{j}\right) \leq 0
$$

Hence,

$$
g_{j}\left(\widehat{x}, \bar{v}_{j}\right) \leq 0=g_{j}\left(\hat{x}, \bar{v}_{j}\right)
$$

Because $(f, g)$ is a pseudoquasi-type-I function at $\bar{x}$, combining equation (70) with equation (72), we conclude that for $\bar{\xi}_{i} \in \partial f_{i}(\bar{x}), \quad i=1, \ldots, l, b \in \mathbb{B}, \bar{\eta}_{j} \in \partial_{x} g_{j}\left(\bar{x}, \bar{v}_{j}\right)$, $\bar{v}_{j} \in \mathscr{V}_{j}(\bar{x}), j=1, \ldots, m$, there exists $u \in \mathbb{R}^{n}$, such that

$$
\begin{gathered}
\left\langle\bar{\xi}_{i}, u\right\rangle+\sqrt{\varepsilon_{i}}\langle b, u\rangle<0, \quad i=1, \ldots, l, \\
\left\langle\bar{\eta}_{j}, u\right\rangle \leq 0, \quad \mu_{j} \neq 0 .
\end{gathered}
$$

Noticing that $\bar{\lambda} \in \mathbb{R}_{+}^{l} \backslash\{0\}$, it holds that

$$
\sum_{i=1}^{l} \bar{\lambda}_{i}\left(\bar{\xi}_{i}+\sqrt{\varepsilon_{i}} b, u\right)+\sum_{j=1}^{m} \bar{\mu}_{j}\left\langle\bar{\eta}_{j}, u\right\rangle<0,
$$

which contradicts to equation (74). Therefore, $\bar{x}$ is a robust $\mathcal{E}$-quasi weak efficient solution of the problem (NUMP).

By the similar arguments, we can prove (ii).

Finally, as the end of this article, we give a concrete example to verify Theorem 3 .

Example 2. Consider the following nonsmooth robust multiobjective programming (NRMP $)_{0}$ problem:

$$
(\mathrm{NRMP})_{0} \begin{cases}\min & f=\left(f_{1}(x), f_{2}(x)\right), \\ \text { s.t. } & g(x, v) \leq 0, v \in \mathscr{V}=[0,1],\end{cases}
$$

where

$$
\begin{aligned}
& f_{1}(x)= \begin{cases}\frac{5}{2} x, & \text { if } x \geq 0, \\
x, & \text { if } x<0,\end{cases} \\
& f_{2}(x)= \begin{cases}\frac{5}{3} x, & \text { if } x \geq 0, \\
x, & \text { if } x<0,\end{cases}
\end{aligned}
$$

and $g: \mathbb{R} \times \mathscr{V} \longrightarrow \mathbb{R}$ is given by

$$
\begin{aligned}
& g(x, v)=v x^{2}, x \in \mathbb{R}, v \in \mathscr{V} \backslash\{0\}, \\
& g(x, 0)= \begin{cases}-\frac{1}{3} x, & \text { if } x<0, \\
-x, & \text { if } x \geq 0 .\end{cases}
\end{aligned}
$$

Taking $\bar{x}=0$ and $\sqrt{\varepsilon}=\left(\sqrt{\varepsilon}_{1}, \sqrt{\varepsilon}_{2}\right)=(1,1)$, it is obvious to get that $\bar{x}=0$ is a robust $\varepsilon$-quasi weak efficient solution of the problem $(\mathrm{NRMP})_{0}$. It is easy to know that

$$
\begin{aligned}
\partial f_{1}(\bar{x}) & =\left[1, \frac{5}{2}\right], \\
\partial f_{2}(\bar{x}) & =\left[1, \frac{5}{3}\right], \\
\partial_{x} g(\bar{x}, v) & =\{0\}, \quad v \in \mathscr{V} \backslash\{0\}, \\
\partial_{x} g(\bar{x}, 0) & =\left[-1,-\frac{1}{3}\right], \mathscr{V}(\bar{x})=[0,1] .
\end{aligned}
$$

For any $\quad x \in \mathbb{R}, \xi_{i} \in \partial f_{i}(\bar{x}), i=1,2, b \in \mathbb{B}$, $\eta \in \partial_{x} g(\bar{x}, 0)$, and $v \in \mathscr{V}(\bar{x})$, there exists $0 \leq u \in \mathbb{R}$, such that

$$
\begin{aligned}
&\left\langle\xi_{1}, u\right\rangle+\sqrt{\varepsilon_{1}}\langle b, u\rangle=\left\langle\xi_{1}, u\right\rangle+\langle b, u\rangle \geq 0 \Longrightarrow f_{1}(x)-f_{1}(\bar{x}) \\
&+\sqrt{\varepsilon_{1}}\|x-\bar{x}\|=f_{1}(x)+\|x\| \geq 0, \\
&\left\langle\xi_{2}, u\right\rangle+\sqrt{\varepsilon_{2}}\langle b, u\rangle=\left\langle\xi_{2}, u\right\rangle+\langle b, u\rangle \geq 0 \Longrightarrow f_{2}(x)-f_{2}(\bar{x}) \\
&+\sqrt{\varepsilon_{2}}\|x-\bar{x}\|=f_{2}(x)+\|x\| \geq 0, \\
& g(x, v) \leq g(\bar{x}, v) \Longrightarrow 0 \cdot u \leq 0, \quad v \in \mathscr{V} \backslash\{0\}, \\
& g(x, 0) \leq g(\bar{x}, 0) \Longrightarrow \eta \cdot u \leq 0, \quad v=0 .
\end{aligned}
$$

It is clear that $(f, g)$ is a pseudoquasi-type-I function at $\bar{x}$. Equations (58) and (59) hold for $\lambda_{1}=1, \lambda_{2}=0, \mu=0$, and $b=-1$. It yields from Theorem 3 that $\bar{x}$ is a $\varepsilon$-quasi weak efficient solution of the problem $(\mathrm{NRMP})_{0}$.

\section{Conclusions}

The optimality conditions of the robust approximate quasi weak efficient solution to a nonsmooth uncertain 
multiobjective programming problem (NUMP) are studied by using the robust optimization method in this note. Firstly, we have introduced $(f, g)$-pseudoquasi-type-I functions to the problem (NUMP), and an example is presented to illustrate its existence. Secondly, under the assumptions that the problem (NUMP) satisfies the extended nonsmooth Mangasarian-Fromovitz constrained qualification and pseudoquasi-type-I convexity, optimality conditions of the robust $\varepsilon$-quasi weak efficient solution are proved.

\section{Data Availability}

The data used to support the findings of this study are included within the article.

\section{Conflicts of Interest}

The authors declare that they have no conflicts of interest.

\section{Authors' Contributions}

All authors contributed equally to the writing of this paper. All authors read and approved the final manuscript.

\section{Acknowledgments}

This research was supported by the Natural Science Foundation of China, under Grant no. 11861002; Natural Science Foundation of Ningxia, under Grant no. NZ17112; Key Project of North Minzu University, under Grant no. ZDZX201804; Nonlinear analysis and financial optimization research center of North Minzu University.

\section{References}

[1] A. Ben-Tal, L. E. Ghaoui, and A. Nemirovski, Robust Optimization, Princeton University Press, Princeton, NY, USA, 2009.

[2] T. D. Chuong and D. S. Kim, "A class of nonsmooth fractional multiobjective optimization problems," Annals of Operations Research, vol. 244, no. 2, pp. 367-383, 2016.

[3] M. Fakhar, M. R. Mahyarinia, and J. Zafarani, "On approximate solutions for nonsmooth robust multiobjective optimization problems," Optimization, vol. 150, pp. 1-31, 2019.

[4] J. H. Lee and L. Jiao, "On quasi $\epsilon$-solution for robust convex optimization problems," Optimization Letters, vol. 10, pp. 1-14, 2016.

[5] X. K. Sun, "Characterizations of robust solution for convex optimization problems with data uncertainty," Acta Mathematica Scientia, vol. 37A, no. 2, pp. 257-264, 2017.

[6] X. K. Sun, X. B. Li, X. J. Long et al., "On robust approximate optimal solutions for uncertain convex optimization and applications to multiobjective optimization," Pacific Journal of Optimization, vol. 13, no. 4, pp. 621-643, 2017.

[7] G. M. Lee and P. T. Son, "On nonsmooth optimality theorems for robust optimization problems," Bulletin of the Korean Mathematical Society, vol. 51, no. 1, pp. 287-301, 2014.

[8] M. Fakhar, M. R. Mahyarinia, and J. Zafarani, "On nonsmooth robust multiobjective optimization under generalized convexity with applications to portfolio optimization," European Journal of Operational Research, vol. 265, no. 1, pp. 39-48, 2018.
[9] F. H. Clarke, Optimization and Nonsmooth Analysis, WileyInterscience, New York, NY, USA, 1983.

[10] O. L. Mangasarian, Nonlinear Programming, McGraw-Hill, New York, NY, USA, 1969.

[11] J. P. Aubin and I. V. Ekeland, Applied Nonlinear Analysis, John Wiley \& Sons, 1984. 